\title{
CALCULATION OF THE STEADY FLOW OF GROUND WATER IN VERTICAL CROSS SECTIONS
}

\author{
L. F. ERNST \\ Agricultural Experiment Station and Institute for Soil Research, \\ Groningen, The Netherlands
}

\section{SUMMARY}

The usual formulae for the relation between rainfall and hydraulic head are only valid for symmetrical, one-layer problems. By introducing horizontal, vertical and radial resistances in the soil profile, it was found possible to obtain solutions to problems of ground water movement in systems with only one water table under asymmetrical conditions and in soil profiles consisting of layers with widely different permeabilities. In this way the ground water flow can be calculated by means of a number of linear equations. Owing to the constant resistances, in some simple cases a linear relation is found between hydraulic head and rainfall or run-off. In the case of a compound drainage system (ditches and tile drains, rivers and secondary watercourses) the relation is represented by a broken straight line. A similar, non-linear, relation is found in the case of a thin aquifer with an elliptic phreatic surface, according to Rothe's formula. An important feature of the method is the characterization of open watercourses by a single value : the radial resistance.

1 The flow of ground water is dependent on the properties of climate, plant growth, soil and topographic conditions. If we take into account only steady states, we have to replace the influence of climate and plants by the very simple equation $N=$ constant. ( $N=$ precipitation - evaporation; mean value of $N>0$, for subirrigation in dry periods $N<0$ ). This assumption is not entirely correct, but it is very useful in agricultural engineering practice. By restricting our attention to vertical cross-sections of the soil, we have eliminated one of the three dimensions of space: all the details of the flow may be represented in a flat plane. In most publications by other workers on this subject (e.g. Rothe, 1924; Hooghoud, 1937, 1940), still more simplifying conditions are assumed': a homogeneous soil, constant levels and distances for parallel open waterways, etc. Special attention has often been paid to the shape of the phreatic surface (Gustarsson, 1946), which might be considered however as a property of minor importance. If we ignore the influence of the shape of the phreatic surface, and make use only of Darcy's law, it appears to be possible to evolve a simple and sufficiently accurate method, in which heterogeneous soils and topographic conditions can be taken into account.

2 The soil is one of the two factors which are really independent of time. Nearly every soil is heterogeneous, often both in the horizontal and in the vertical direction. We might distinguish several horizontal layers, of successively great and slight permeability. If the ratio between the great and the slight permeabilities is 1:10 or more (DE GLEE, 1930), we may suppose that the flow in the well-conducting layers is horizontal, in the poorly conducting layers vertical. In the case of smaller ratios we consider the soil to be homogeneous and make use of the mean permeability.

3 The topographic conditions cause certain variations in the distances $L_{\mathrm{i}}$. the wet contours $u_{i}$ and the levels $h_{o, i}$ of the open waterways. Any given problem in which the variations mentioned are involved is solvable by means of a simple 
line diagram (e.g. Fig. 5 for a one-layer problem), provided a) the superimposing of several currents is allowed and b) the horizontal dimensions in the problem exceed the vertical dimensions. The justification of such a simplification, and of the two necessary conditions, will be considered under 4 and 5 .

4 Because we make use of superimposing, the shape of the phreatic surface is not allowed to have a sensible influence on the value of the resistances. So the maximum fall $\Delta h$ of the phreatic surface must be small in proportion to the mean thickness of the aquifer : $\triangle h<1 \% \bar{D}$.

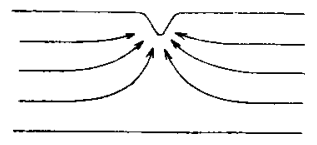

FIG. I

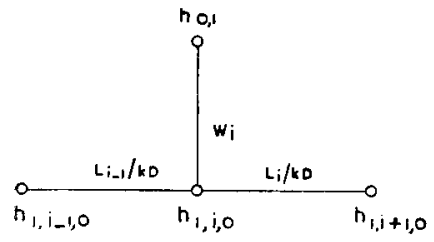

FIG. 4

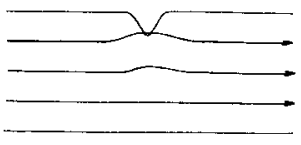

FIG. 2

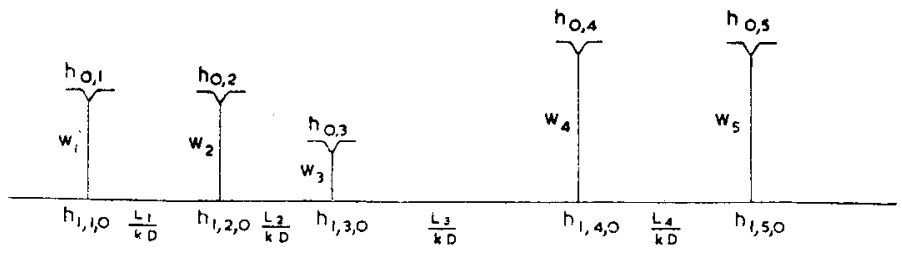

FIG. 5

From Fig. 1, 2 and 3 it is evident that superimposing is permissible in the vicinity of open waterways (also valid for more layers). The symmetrical flow in Fig. 1 may be replaced approximately by the scheme in Fig. 4. As the flow in Fig. 2 is practically horizontal, the horizontal line in Fig. 4 would be sufficient in this case. Addition of Fig. 1 and 2, both multiplied by a suitable constant, will give any arbitrary distribution of the components of the flow (e.g., Fig. 3). Use of a line diagram like that in Fig. 4 is therefore permissible in every case.

We introduce horizontal resistances $L_{i} / k D$ (see horizontal line sections in Fig. 4 and 5) and radial resistances $w_{i}$ (vertical line sections). These resistances are measured in days/meter. This system is also valid in the case of more layers. Badly permeable layers must be characterized by the vertical resistance $c=D / k$. The dimension of the vertical resistances is "time".

5 In one-layer problems, where the conditions $\Delta h<1 / 2 \bar{D}$ and $N=0$ are satisfied, a linear relation exists between hydraulic head $h$ and horizontal coordinate: the phreatic surface is a flat surface. When $N=$ constant $\neq 0$, the phreatic surface has a parabolic shape. Both when $N=0$ and $N \neq 0$, the shape is different in the vicinity of an open waterway. A sufficient approximation is only valid at a distance $D$ from the open water. So we have as a second necessary condition: $D<1 / 2 L$.

A similar condition is required in a case in which a layer of poor permeability covers a layer of good permeability: $\Sigma D_{j}<\frac{1}{2} \mathrm{~L}$. Equations are little different from those of the one-layer problem. In the case of two layers with a high permeability, separated by a layer with a low permeability, equations 
are much more complicated. In the equations for the hydraulic head $h$ and the horizontal intensity $q$ of the flow we shall find linear, square and exponential terms. $\Delta h$ must be smaller than half the mean value of the upper layer.

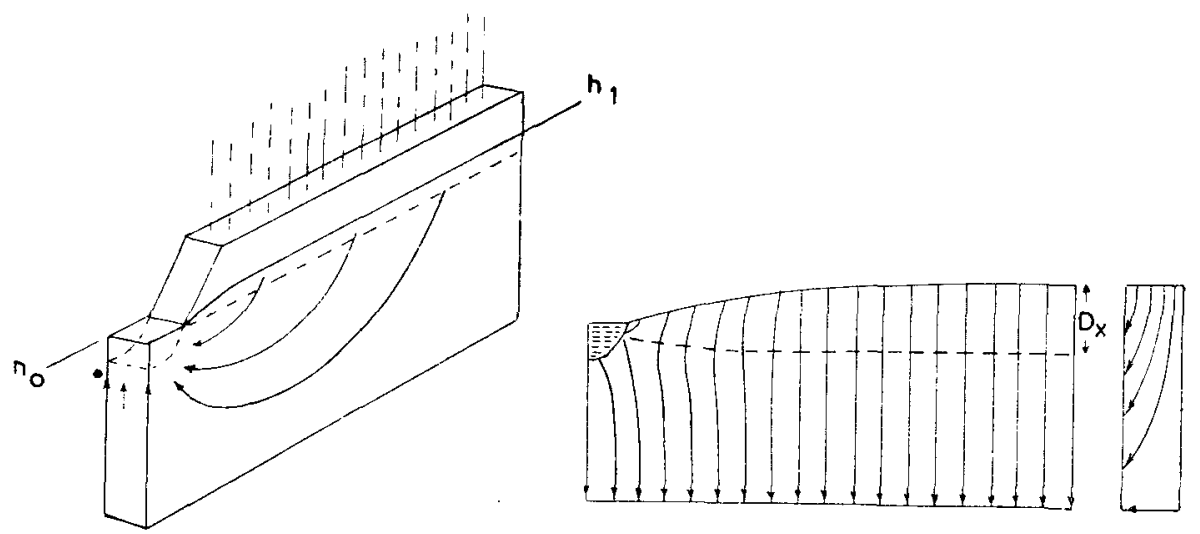

Fig. 6. Symmetrical Drainage of ground WATER,

Fig. 7. Vertical component.

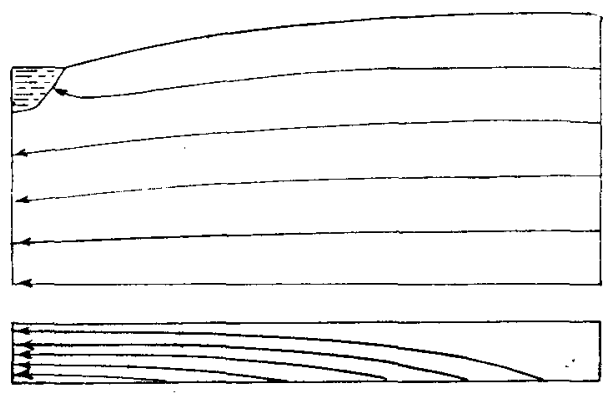

Fig. 8. Honizontal Component.

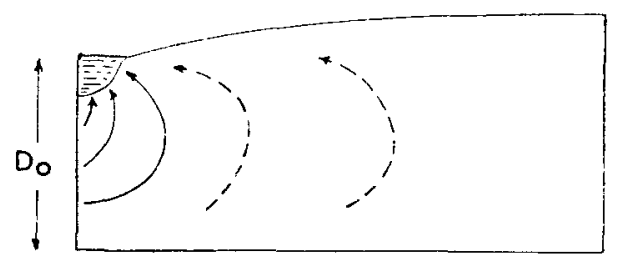

Fig. 9. Radial component.

In all these cases we might consider that only $h$ and $q$ are the unknown variables. In this way linear terms remain. Irrespective of the number of layers, we can reduce every problem to the solution of a number of linear equations.

6 The following equations are valid in symmetrical situations. "Symmetrical situations" are those in which all $L, D, w, u$ and $h_{0}$ have the same value. $h_{1}$ and $h_{0}$ are the maximum and minimum value of $h$. The two conditions mentioned under 4 and 5 must again be satisfied.

One layer: $\quad h_{1}-h_{0}=\frac{N L^{2}}{8 k D}+\frac{N L}{\pi k} \ln \frac{D_{0}}{u}$

Two layers: $\quad h_{1}-h_{0}=N \frac{D_{x}}{k_{1}}+\frac{N L^{2}}{8\left(k_{1} D_{1}+k_{2} D_{2}\right)}+N L w$

Fig. 6, 7, 8 and 9 illustrate the derivation of equations (1) and (2). In eq. (1) the small term $N D_{x} / k$ is ignored. We have substituted for the radial resistance $w$ an expression giving a good approximation for $u<D_{0}$. For larger values of $u$ this term approaches zero and may even have a small negative value. 
In the case of three and four layers (the bottom layers having relatively high permeability) equations (3) and (4) are valid. In eq. (3) the same expression for the radial resistance is used as in eq. (1). This expression may also be used in eq. (4), in those cases in which open waterways cut into the second layer.

$h_{1}-h_{0}=\frac{N c_{2} a_{1,2}}{8\left(a_{1,2}+a_{3,2}\right)}\left\{a_{3,2} L^{2}+\frac{4 a_{1,2} L}{\sqrt{a_{1,2}+a_{3,2}}} \operatorname{tgh} \frac{L}{4} \sqrt{a_{1,2}+a_{3,2}}\right\}+\frac{N L}{\pi k_{1}} \ln \frac{D_{0}}{u}$ (3)
$h_{1}-h_{0}=N \frac{D x}{k_{1}}+\frac{N c_{3} a_{2,3}}{8\left(a_{2,3}+a_{4,3}\right)}\left\{a_{4,3} L^{2}+\frac{4 a_{2,3} L}{\sqrt{a_{2,3}+a_{4,3}}} \operatorname{tgh}{ }_{4}^{L} V a_{2,3}+a_{4,3}\right\}+N L w$

Abbreviation : $a_{i, j}=k_{i} \frac{1}{D_{i} c_{j}}$

Among the symmetrical one-layer problems, in which at least one of the two conditions mentioned is not satisfied, we have two special cases with a simple solution.

Eq. (5) is derived for an aquifer of infinite thickness. Eq. (5) gives a very good approximation for $D>0.5 L$ and even for $D>0.25 L$ the approximation might be considered as sufficient. The wet surface $u$ and the drop in hydraulic head $h_{1}-h_{0}$ must be smaller than $0.2 L$.

$$
h_{1}-h_{0}=\frac{N L}{\pi k} \ln \frac{L}{u}
$$

In the case of a shallow impermeable layer, we have to take into account the variable thickness $D$. We take the height of the water table with reference to the impermeable layer: $h=D$. When $h_{1}-h_{0}>1 / 2 \bar{D}$ and $\bar{D}<1 / 2 L$, eq. (6) is valid.

$$
h_{1}^{2}-\left(h_{0}+N L w\right)^{2}=\frac{N L^{2}}{4 k}
$$

Substitution of $w=0$ in eq. (6) changes it into eq. (7) (Rothe, 1924).

$$
h_{1}{ }^{2}-h_{0}{ }^{2}=\frac{N L^{2}}{4 k}
$$

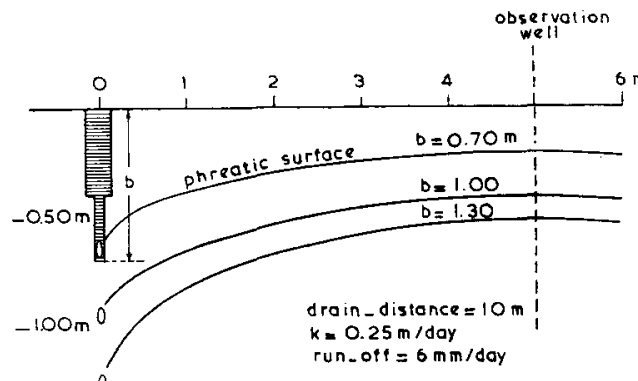

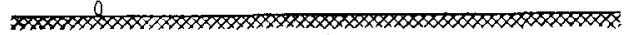
imper meable layer

FIG. 10

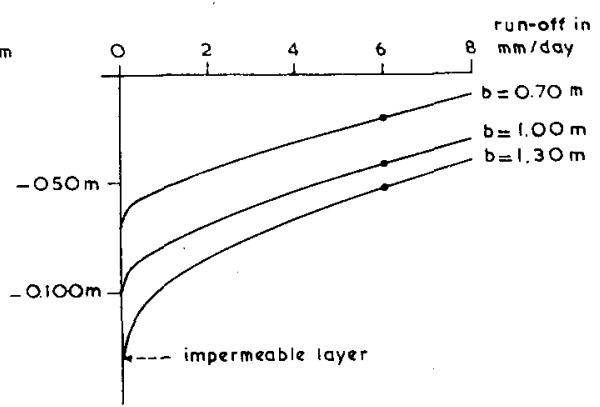

hydraulic head

in observation well
Fig. 11 
For eq. (6) and (7), superimposing is not allowed. The difference between them and eq. (1) is however rather small. In many cases the strongest curvature is caused by the radial resistance. The second term of equation (1) applies to a parabolic water table; eq. (6) and (7) follow from an elliptic water table. Substitution of $\bar{D}=1 / 2\left(D_{\min }+D_{\max }\right)=\frac{1 / 2}{12}\left(h_{0}+N L w+h_{1}\right)$ as a mean value of the thickness of the aquifer changes eq. (6) into eq. (1).

7 It follows from 4 and 5 that every asymmetrical problem may be solved by means of a number of linear equations. Generally speaking, it is possible to reduce the problem to about $2 m n$ equations, $m$ being the number of horizontal layers of high permeability and $n$ the number of open watercourses.
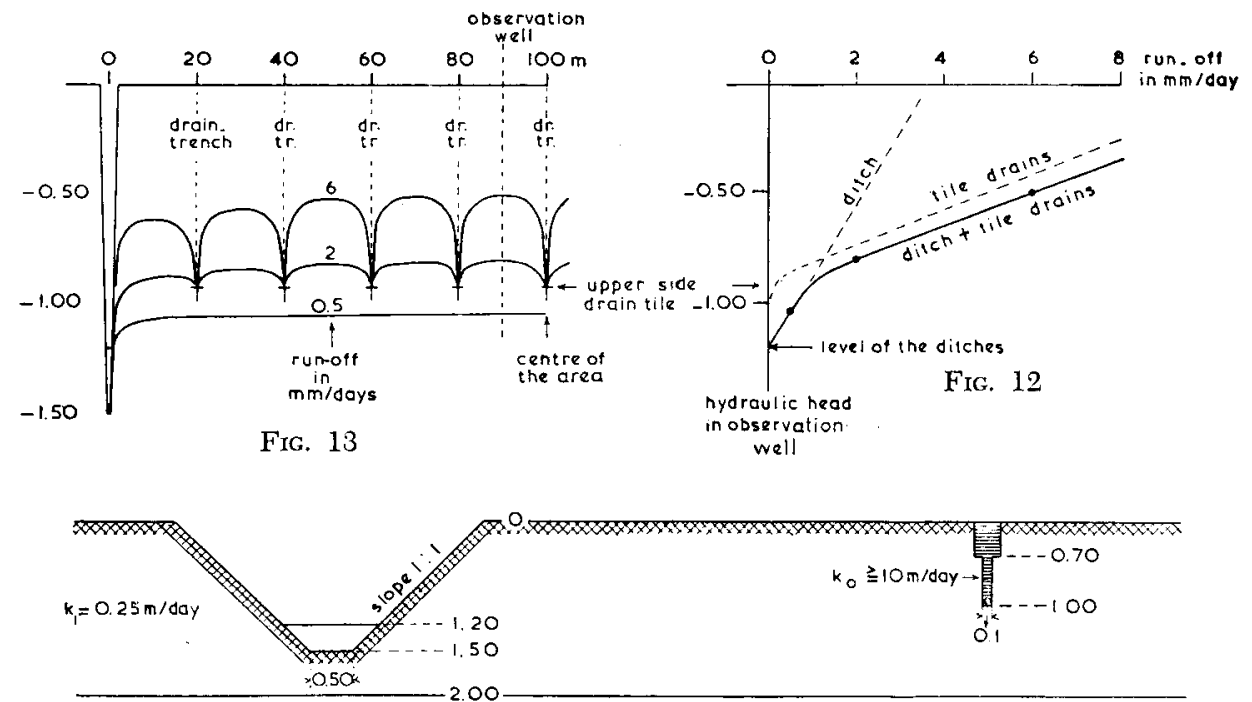

$k_{2}=5 \mathrm{~m} / d 0 y$

$D_{2}=15 \mathrm{~m}$

FIG. $12 \mathrm{a}$

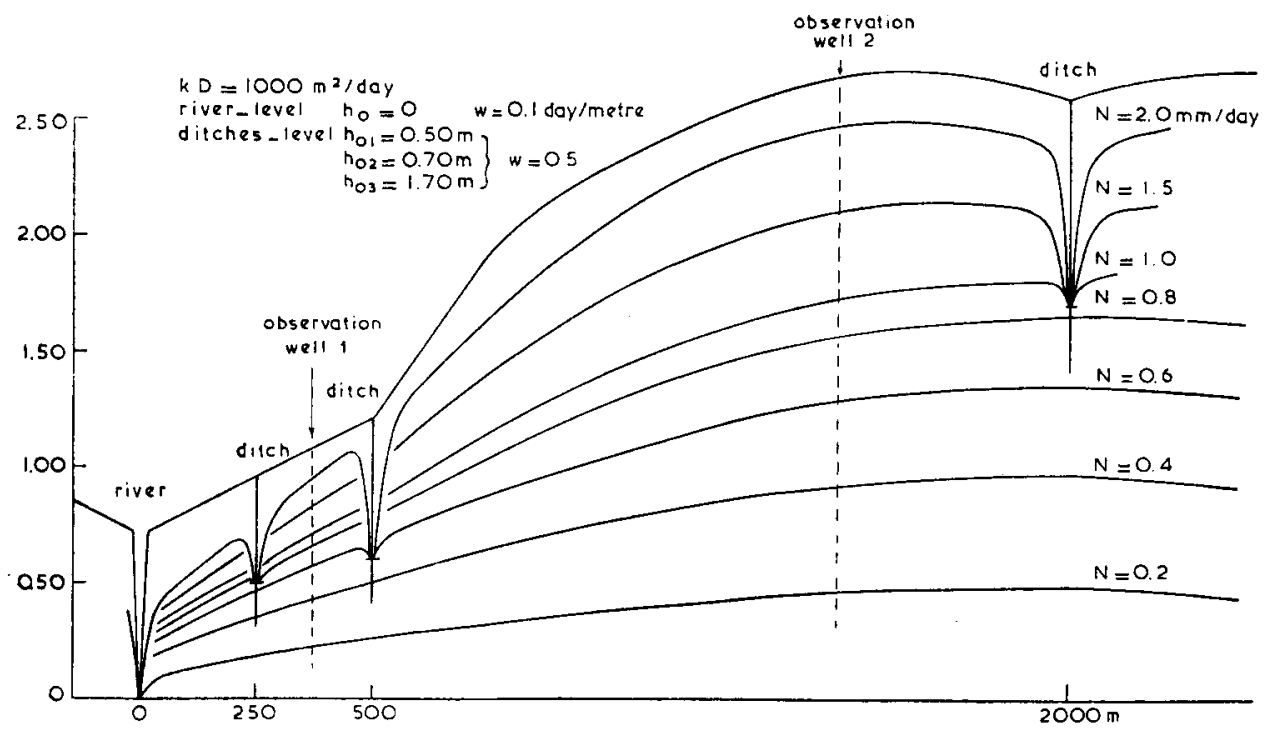

FIG. 14 
For one and two-layer problems with $N=$ constant $\neq 0$, we prefer the following indirect method for solution of the linear equations. First we assume that for the right value of $N$ the situation is symmetrical in every strip between two open waterways. A second calculation has to be carried out for $N=0$, and in this case we have to substitute for $h_{o, i}$ values which are such that their addition to the first calculation will give the right values for $h_{o, i}$.

Fig. 10,12 and 14 show the shape of the phreatic surface as calculated for several symmetrical and asymmetrical situations. Whereas we can deduce from eqs. (1) to (5) that the proportion $\frac{h_{1}-h_{0}}{N}$ has to be constant, it follows from Fig. 11, 13 and 15, that this is not always the case. In Fig. 11 this discrepancy is caused by the small value of $D$. In Fig. 13 and 15, as in nearly all compound drainage systems, it follows from the fact that constant levels in open watercourses are an exception. The gradual drop of the water table causes tile drains and little ditches to become dry during a more or less large part of the summer.

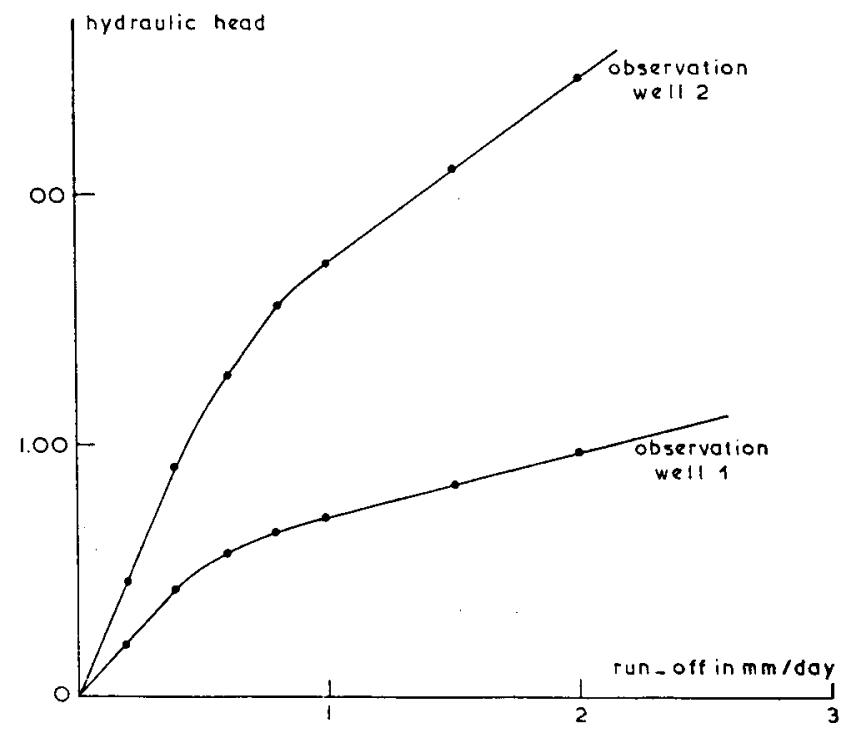

FIG. 15

\section{BiBLIOGRAPHY}

GLEE, G. J. DE : Over grondwaterstromingen bij wateronttrekking door middel van putten, Delft, 1930.

Gustafsson, Y.: Untersuchungen über die Strömungsverhältnisse in gedräntem Boden. Acta Agr. Suecana, 2 (1946) 1-157.

Hooghoudt, S. B. : Versl. Landbouwk. Onderz. 43 (13) B (1937). Versl. Landbouwk. Onderz. 46 (14) B (1940).

Rothe, J.: Die Strangentfernung bei Dränungen. Landwirtsch. Jahrb. 59 (1924) 453-490. 